\title{
La construcción social de la realidad: el papel de los profesionales en el establecimiento de la agenda temática
}

\author{
María Dolores CÁCEREs ZAPATERO \\ Universidad Complutense de Madrid \\ caceres@ccinf.ucm.es
}

Recibido: $13 / 12 / 2010$

Aceptado: 22/07/2011

\begin{abstract}
Resumen
Esta colaboración presenta los resultados de un estudio Delphi, como parte de una investigación más amplia cuyo objeto ha sido el análisis del discurso sobre la comunicación social convertido en tema de agenda en la prensa, y los ejes sobre los cuales se hace hegemónico dicho discurso a propósito de "la verdad" y "la comunicación". En este artículo se aborda cómo las prácticas productivas y rutinas profesionales del periodismo afectan a la construcción de los discursos hegemónicos en la sociedad, desde la voz de sus propios productores: los periodistas de fuentes y los periodistas de medios. Se plantea, por una parte, el flujo de informaciones entre las fuentes y los medios y, por otra, los hábitos y actitudes que comprometen dicho tráfico.
\end{abstract}

Palabras clave: establecimiento de agenda, sociología de los emisores, rutinas periodísticas, producción de la noticia.

The social construction of reality: the role of professionals in the thematic agenda building

\begin{abstract}
This paper presents the results of a Delphi study, as part of a wider investigation, whose mind is to analyze the discourse of social communication that become the subject of the news agenda, and de foundations on which this discourse becomes hegemonic, about "truth" and "communication". This article discusses how the production practices of journalism and professional routines affect the construction of hegemonic discourses in society, from the voice of their own producers: journalists of sources and journalists of media This raises one hand, the flow of information between the sources and media and, second, habits and attitudes that compromise this traffic.
\end{abstract}

Keywords: agenda building, newsmaking, newspaper routines, production of news.

\section{Referencia normalizada}

CÁCERES ZAPATERO, María Dolores (2011): "La construcción social de la realidad: el papel de los profesionales en el establecimiento de la agenda temática". Estudios sobre el mensaje periodístico. Vol. 17, núm. 2, págs.: 303-324. Madrid, Servicio de Publicaciones de la Universidad Complutense.

Sumario: 1. Introducción. 2. Objetivos y metodología. 3. Flujo de informaciones entre fuentes y medios. 3.1. Herramientas de contacto preferibles. 3.2. Volumen de respuesta a las demandas de contacto entre periodistas de fuentes y medios. 3.3. Interés de las noticias. 4. Valoraciones y apreciaciones sobre las relaciones entre fuentes y medios. 4.1. Valoración de las relaciones entre los gabinetes de prensa y los medios. 5. Prácticas profesionales y rutinas de selección, jerarquización y ubicación de informaciones. 5.1. Selección. 5.2. Jerarquización. 5.3. Ubicación. 6. Credibilidad de la fuente y otros criterios que comprometen las rutinas profesionales. 7. Afinidades ideológicas, políticas y económicas en la valoración de las relaciones entre fuentes y medios. 8. Hábitos y actitudes que comprometen las prácticas profesionales. 8.1. Preferencia y uso de géneros periodísticos. 8.2. Autoría y protagonismo de las informaciones publicadas como consecuencia de las relaciones entre periodistas de fuentes y medios. 8.3. Elección de las secciones en las cuales se ubican las informaciones publicadas. 8.4. Estructura del discurso en la que se integra la información de fuentes. 9. Conclusiones. 10. Referencias bibliográficas. 


\section{Introducción}

Hoy es generalmente aceptado que aquello que publican los medios a propósito del acontecer social se torna en la realidad destinada a ser conocida, y que aquello que carece de visibilidad pública no tiene existencia para las audiencias. Se genera así una segunda realidad superpuesta al acontecer de referencia que adquiere legitimidad en el discurso de los medios y existencia autónoma respecto a los hechos, pero que acaba comprometiendo a todos los actores sociales. El empeño de los medios se centra en hacerse transparentes en su tarea de informar y presentar su labor de mediación ante la opinión pública, como una actuación neutra que no afecta a la visión de la realidad que transmiten, si bien hoy es comúnmente aceptado que los medios tienen capacidad para estructurar los conocimientos de la audiencia, lo que ha sido definido como la función de establecer agenda (McComBS, M., 1996). Los medios se presentan a sí mismos ante la sociedad, como una instancia meramente técnica, es decir como un vehículo que hace llegar a la audiencia los hechos noticiosos solo desde criterios de relevancia periodística (newsvalues), defendiendo su profesionalidad y ocultando su labor de mediación y, por consiguiente, de construcción (TuchmAN, G., 1993). En esta tarea mediadora se hace presente su labor profesional como conjunto de prácticas que afectan a la selección, jerarquización y ubicación de las noticias, además de a otros aspectos relacionados con el tratamiento y enfoque (framing).

Habida cuenta de que la producción de comunicación dentro de la industria mediática responde a un saber hacer de los profesionales, analizar el discurso mediático que guarda referencia con la propia actividad de los medios de comunicación y sus condiciones de producción, constituye un objetivo estratégico. Esta colaboración presenta los resultados de un estudio Delphi, como parte de una investigación más amplia cuyo objeto ha sido el análisis del discurso sobre la comunicación social convertido en tema de agenda en la prensa, dentro del proyecto de investigación I+D+i: "El discurso hegemónico a propósito de la "verdad" y la "comunicación": lo que dicen los Medios de Comunicación social sobre la comunicación social" (Ref. SEJ2007-62202SOCI), dirigido por José Luís Piñuel. Dicha investigación, que se propone desvelar los ejes sobre los cuales se hace hegemónico el discurso de la prensa a propósito de "la verdad" y "la comunicación", se ha llevado a cabo a partir de un Análisis de contenido de los siete principales diarios españoles (El País, El Mundo, ABC, La Vanguardia, La Razón, El Correo y La Voz de Galicia) cuyo discurso se contrasta con el producido por jefes de prensa y directores de comunicación de diferentes tipos de organizaciones: empresas, administración, instituciones, partidos políticos y sindicatos, que aquí se expone.

\section{Objetivos y metodología}

Este trabajo plantea los resultados de una parte de la citada investigación realizada a partir de la técnica Delphi, en la que se exploran las prácticas productivas y rutinas profesionales del periodismo que afectan a la construcción de los discursos hegemónicos en la sociedad en un momento histórico. Se trata de mostrar rutinas de trabajo e influencias no siempre explícitas que afectan a la producción de las noticias, desde la voz de sus propios productores: los periodistas de fuentes y los periodistas de medios. En este sentido, se abordan aspectos que se relacionan con la construcción de la agenda mediática, la actividad de gatekeeping y los condicionantes de la producción de noticias. 
A diferencia del mundo anglosajón, en el ámbito hispanohablante no abundan los trabajos de tipo cualitativo (FrankenBerg, L. y LozANo, J.C, 2010; HumANES, M.L., 2007) abordados desde técnicas observacionales o conversacionales. En este caso, se ha optado por la técnica Delphi que permite obtener un discurso construido conjuntamente por expertos en el ámbito que se desea conocer, evitando las distorsiones producidas en las discusiones de grupos, como las influencias de unos sujetos sobre otros o los efectos de la adhesión a la opinión mayoritaria (GAITÁn, J.A. y PIÑUEL, J.L., 1998). Esto ha permitido llegar a acuerdos relativos sobre los temas planteados, a partir de aproximaciones sucesivas y minimizando los efectos de la interacción, sin sacrificar opiniones importantes e información esencial.

Los objetivos del Delphi se centran en dos ejes temáticos esenciales que han sido abordados con profundidad y extensión, en tres rondas sucesivas, a partir de una decena de preguntas en cada una de ellas. A partir de este sistema de preguntas y re-preguntas, ha sido posible clarificar y decantar las posturas de los profesionales respecto a las cuestiones planteadas en cada uno de estos dos ejes:

- Eje 1: Flujo de informaciones entre las fuentes y los medios: se aborda cómo se establecen las relaciones entre los gabinetes de prensa y los medios y cuáles son los condicionantes que operan en estas relaciones.

- Eje 2: Hábitos y actitudes que comprometen el flujo de informaciones entre fuentes y medios: se aborda cómo se desempeñan habitualmente las prácticas profesionales y rutinas productivas y cómo afectan a la construcción de las noticias.

El Delphi fue lanzado entre el mes de noviembre de 2008 y el mes de marzo de 2010 a 20 expertos, de los cuáles respondieron 17. El cuestionario fue enviado y contestado por correo electrónico, evitando así el sesgo presencial por parte de aplicadores y expertos colaboradores. Se elaboraron dos cuestionarios ligeramente diferentes en función de que fueran dirigidos a periodistas pertenecientes a los gabinetes de comunicación de empresas e instituciones, o destinados a periodistas de medios y agencias de noticias.

\begin{tabular}{|c|}
\hline Ficha técnica \\
\hline - Técnica aplicada: cuestionario Delphi \\
- Medio de realización: correo electrónico \\
- Universo: periodistas de fuentes y periodistas \\
de medios ${ }^{1}$ \\
- Tamaño de la muestra: 17 expertos \\
- Fecha del trabajo de campo: \\
- Primera Ronda: noviembre-diciembre 2008 \\
- Segunda Ronda: julio-septiembre 2009 \\
- Tercera Ronda: marzo-abril 2010 \\
- Fuentes representadas \\
- Organizaciones patronal y sindicales \\
- Instituciones políticas del Estado y del ám- \\
bito de la cultura \\
- Empresas de distintos sectores: auditoría y \\
consultoría, hipermercados, tecnología, \\
comunicación, transportes y juego \\
- Medios representados \\
- Un periódico de difusión nacional \\
- Dos agencias de noticias \\
\hline
\end{tabular}

${ }^{1}$ La relación de expertos no aparece haciendo caso de los requerimientos de los informantes, quienes solicitaron expresamente, en algunos casos, no aparecer vinculados a su institución; otros sin embargo no mostraron inconveniente. A fin de dar homogeneidad al repertorio de expertos, no se han consignados los nombres. Si algún lector está muy interesado, los autores de esta investigación facilitarán la identidad para ser comunicada, aunque no publicada. 


\section{Flujo de informaciones entre fuentes y medios}

Con la proliferación de los gabinetes de prensa en el periodismo actual, frecuentemente es la fuente la que hace la noticia, dando lugar a lo que ha dado en llamarse información convocada. Trabajos anteriores ponen de manifiesto (CANEL et al., 2000) que uno de cada tres periodistas piensa que la agenda de noticias está determinada por convocatorias oficiales o institucionales más que por la propia iniciativa de los redactores y que éstos se limitan a editar las notas de prensa o los comunicados que las fuentes les hacen llegar, para adaptarlos al espacio disponible.

Respecto a cómo se lleva a cabo el flujo de informaciones entre las organizaciones, instituciones o empresas y los medios, interesaba conocer por una parte, cuáles son las vías más habituales a partir de las cuáles se establece el contacto así como cuál es la resonancia o eco que ese tráfico alcanza cuando es publicado. Los datos a propósito de este eje se obtuvieron a partir de las tres preguntas siguientes formuladas a los expertos en la primera ronda del Delphi:

1) “¿Cuáles son los tipos de herramientas preferibles para los contactos con los periodistas (dossieres de prensa, comunicados, convocatorias, encuentros personales, llamadas, e-mails, etc.)?"

2) “¿Qué medios responden más y mejor a sus demandas de contacto?”

3 ) ¿¿Cuál es el tipo de noticias de las que Vds. facilitan a los Medios que tienen más interés para ellos? y cuáles son las noticias que, no siendo facilitadas por Vds. a los Medios, tienen más interés para ellos?"

\subsection{Herramientas de contacto preferibles}

Respecto a cuáles son las herramientas preferibles para los periodistas de fuentes ${ }^{2}$ a la hora de establecer los contactos con los periodistas de medios, las respuestas mostraron que, no solo lo más frecuente es que se lleven a cabo a través de llamadas de teléfono y que cada vez adquieren más importancia las nuevas herramientas de comunicación como el correo electrónico, sino que estos medios se emplean, sobre todo, para sostener las relaciones personales entre el comunicador de fuentes y el comunicador de medios, pero sin descartar también otras alternativas, como la rueda de prensa, cuando el tema lo requiere o respecto a acontecimientos especiales ${ }^{3}$ :

"Yo prefiero comunicarme con los periodistas a través del teléfono, mucho más directo, seguido de los mails donde les envío comunicados y convocatorias".

"Lo más habitual, en el día a día, son los contactos personales (telefónicos) y las notas de prensa".

"Primero, e-mails; segundo, llamadas telefónicas; tercero, encuentros personales".

"También con cierta periodicidad realizamos contactos "tú a tú" con el periodista, en desayunos o comidas de trabajo".

"Periódicamente, y cuando el tema lo requiere, hacemos un convocatoria de Rueda de Prensa".

${ }^{2}$ El periodista de fuente es el comunicador que gestiona el conocimiento de la organización, institución u organismo para el que trabaja.

${ }^{3}$ Se reproducen literalmente porciones del discurso producido por los expertos para ilustrar las afirmaciones que se vierten en este trabajo. 
En segundo lugar, señalaron otras formas de comunicación habituales: comunicados, notas de prensa y convocatorias para encuentros grupales sobre temas específicos:

"[...] las más frecuentes son los comunicados y las convocatorias".

"La más frecuente son las notas de prensa mandadas por correo electrónico y las llamadas para charlar sobre temas o hacer seguimiento de las informaciones".

"El envío de notas y convocatorias es más excepcional y varía en función de la actividad de la empresa. En función de la relevancia del tema que queremos proponer hacemos también convocatorias a todos los medios".

Con el fin de profundizar en estas cuestiones, en la segunda ronda del Delphi, los profesionales fueron de nuevo preguntados acerca de los ítems aparecidos en las respuestas de la primera ronda. Se corroboró que para las fuentes las relaciones personales son lo más importante y que, a ellas, se añaden otras observaciones como el peso o importancia que tiene la fuente y su credibilidad. El flujo de comunicación entre empresas e instituciones y medios, se lleva a cabo preferentemente por las siguientes vías, en este orden:

- Encuentros personales (citados por 14 de 17 expertos como lo más utilizado: diez de ellos afirman utilizarlos mucho y cuatro bastante).

- Llamadas telefónicas (citadas también por 14 de 17 expertos como lo más utilizado: siete de ellos afirman utilizarlo mucho y siete bastante).

- Correo electrónico (citado por 13 de 17 expertos como lo más utilizado: cinco de ellos afirman utilizarlo mucho y ocho bastante).

- Comunicados de prensa (citados por ocho de 17 expertos como lo más utilizado: aunque sólo dos de ellos afirman utilizarlo mucho y seis bastante; dos expertos afirman utilizarlo poco)

- Convocatorias para encuentros grupales (citadas por ocho de 17 expertos: tres afirman utilizarlo mucho y el resto bastante).

En resumen, son los contactos personales los que resultan más valorados ["Por supuesto, para lograr colocar los mensajes es esencial el contacto personal con los medios"] seguidos de las llamadas telefónicas, y el correo electrónico en tercer lugar. Las convocatorias de encuentros y comunicados de prensa, ocupan las siguientes posiciones en el orden de prelación marcado por los profesionales.

Hay que añadir que, en general, los periodistas señalan que, para lograr la mayor presencia en los medios, hay que sumar a las relaciones personales el interés de la noticia, la importancia del tema o la credibilidad de la fuente:

"Las relaciones personales son, sin duda, fundamentales y en muchas ocasiones sí puede decirse que son lo más importante. No obstante, la fuerza que tiene "un buen tema" o la credibilidad que haya adquirido la Institución como fuente informativa suele también imponerse como criterio a la hora de aparecer en los medios"

"Rara vez se "vende" algo que tenga escasa relevancia sólo por la relación que se tenga con los periodistas"

"En lo que sí influye mucho una buena relación es en la imagen que se transmite de la Institución o de la persona que la dirige"

"Una vez establecida una relación de confianza, hay que aportar valor añadido con mensajes y contenidos atractivos para el medio". 
Y también coinciden en que, en todo caso, mantener las mejores relaciones con los periodistas sólo lleva a lograr la mayor presencia en los medios si se respetan compromisos y hay mutuo conocimiento:

"El periodista también tiene sus preferencias y, lógicamente, un mayor conocimiento de la fuente (incluido su representante) le ayudará a poder escribir sobre ese tema con mayor conocimiento".

“[...] los profesionales de los medios diferencian el trato personal y lo que es noticia. No publicarán una información porque se lleve bien con el responsable de prensa, sino porque interesa a su medio. De igual manera, no ocultarán una noticia negativa de una institución por llevarse bien con el responsable de prensa. Los periodistas son profesionales y saben diferenciar".

"Si nos hemos labrado una imagen de fuente veraz que da datos rigurosos y que está disponible para las buenas y las malas noticias, y además la relación personal es cordial, ayudará sin ninguna duda, pero no por la "amistad" sino por la credibilidad que se ha ganado".

"Mantener excelentes relaciones con periodistas facilita estar accesible y poder acceder a ellos para facilitar que el punto de vista de la organización pueda estar presente en sus informaciones".

\subsection{Volumen de respuesta a las demandas de contacto entre periodistas de fuen- tes y medios}

Para conocer cómo se produce el tráfico de informaciones entre fuentes y medios, interesaba saber cómo es la respuesta de los medios a las demandas de los periodistas de fuentes. Así, fueron preguntados a propósito del volumen de esta respuesta y a propósito de qué medios responden más y mejor a sus demandas de contacto. Los responsables de comunicación de las instituciones, organizaciones y empresas contestaron, en general, que el volumen de respuesta es amplio en su mayor parte y además:

"8 de cada 10 responden, es decir puedes mantener un diálogo con ellos, aunque no siempre ese diálogo conlleva resultados para la fuente".

"Tenemos en general una buena respuesta de los medios, especialmente con determinado tipo de noticias".

"La respuesta varía en función de la noticia. Cuanto más relevante, mayor respuesta en forma de llamadas para solicitar más información; mayor número de periodistas asistentes a la rueda de prensa y por consiguiente, mayor cobertura mediática".

Respecto a qué medios responden más se ha detectado, en la primera ronda, una cierta dispersión en las respuestas, siendo la prensa económica y las agencias las que han resultado ser las más comprometidas en publicar las informaciones recibidas, y la prensa generalista la más selectiva.

Por su parte, los profesionales de medios respondieron unánimemente que las fuentes responden de manera satisfactoria a sus requerimientos de información, ya sea para confirmar noticias o ampliar la información, en un juego natural de mutuo interés. 
A partir de estas observaciones, en la segunda ronda del Delphi se definieron con mayor precisión algunos aspectos sobre la relevancia de la noticia, el interés estratégico de la agenda y el compromiso de publicación. Se cuestionó a los profesionales de fuentes, en primer lugar sobre el hecho de corroborar o no, si el perfil del medio resulta determinante en cuanto a un mayor compromiso a la hora de publicar sus informaciones: casi la totalidad de las respuestas (trece de diecisiete) fueron afirmativas:

"Sí es determinante el perfil del medio. Cada medio estudia el tipo de información que puede ser de interés para su cliente y actúa en consecuencia".

"Absolutamente de acuerdo".

"Obviamente, el perfil del medio determina el mayor o menor interés en la noticia".

Es el perfil temático del medio -no ideológico- lo que determina el interés de la información; las fuentes creen que el medio espera así satisfacer a sus audiencias. Ante la pregunta "¿Podría señalar quiénes adquieren mayor compromiso: los medios generalistas, las agencias de noticias o la prensa especializada?", ocho de diecisiete periodistas resaltaron que la prensa especializada adquiere mayor compromiso que los medios generalistas y las agencias de noticias para publicar aquellas informaciones. La agenda temática de esta prensa especializada (económica, deportiva, etc.) se ve reducida al ámbito de su especificidad frente a la generalista o frente a las agencias de noticias que, en principio, deben hacerse eco de todas las informaciones.

En cambio, para los medios el compromiso de publicación puede estar menos garantizado si las fuentes de las noticias son empresariales, en la medida en que los medios consideren que su publicación puede servir a intereses comerciales, como frecuentemente se les ha criticado (ORTEGA, F., 2008: 236).

Cuando se pregunta a los profesionales: “¿Puede corroborar si ese compromiso varía según qué secciones en el mismo medio, en cuanto a ver publicadas en general las informaciones de su organización o empresa?", se obtiene una respuesta afirmativa rotunda en once de los diecisiete periodistas interrogados. La publicación de las informaciones de empresas e instituciones está así condicionada por las secciones temáticas de los medios.

De esta manera, el carácter de las fuentes (v.g. empresarial, político, etc.) hará que los medios tiendan a seleccionar sus informaciones para adscribirlas a determinadas secciones (v.g. Economía, Política, etc.), pero no a otras:

"Creo que la gran mayoría de organizaciones están muy condicionadas a salir en una determinada sección, pero no en otras, salvo circunstancias muy excepcionales".

Esto ocurre siempre, salvo que el interés excepcional de la noticia aconseje que su publicación no se limite a las secciones habituales:

"La tipología propia de nuestras informaciones así como nuestro perfil como Institución, nos circunscriben básicamente a dos de ellas, que funcionan más o menos igual respecto a sus criterios de selección de las informaciones a publicar. Aparecer en otras secciones es excepcional y siempre, en estos casos, responde únicamente al interés de la noticia"

En algún caso, se entiende que la profesionalidad y sensibilidad de los periodistas encargados de esas secciones determina si las informaciones acaban publicándose: 
"Puede variar en función de la profesionalidad o sensibilidad que muestren los responsables de esas secciones"

Los medios en cambio señalan que los intereses que las fuentes tienen en la difusión de sus informaciones, les lleva a optar por no servir a intereses particulares.

\subsection{Interés de las noticias}

Respecto a la pregunta "¿Cuál es el tipo de noticias de las que Vds. facilitan a los Medios que tienen más interés para ellos?" se obtuvieron respuestas muy diversas dependiendo de la fuente de que se trate en cada caso. Por ello, el perfil del medio resulta determinante a la hora de mostrar interés, según las fuentes:

"Según he podido compartir con colegas, el perfil de los medios es determinante a la hora de anticipar su interés. Los periódicos y medios económicos son más accesibles a todas las informaciones provenientes de empresas, mientras que los medios de información general son mucho más selectivos (dedican menos espacio a la información empresarial)"

"Depende del tipo de prensa (diaria, económica, femenina, life style, radio, televisión...)"

En cuanto a aquellas noticias no facilitadas por las fuentes y que más demandan los medios, las respuestas apuntaron en dos direcciones: por una parte, la demanda de temas conflictivos, llamativos, situaciones de riesgo, asuntos polémicos y datos financieros y cambios de directivos de las organizaciones:

"En general, si el asunto es polémico, alarmante, muy llamativo, la petición de información u opinión por parte de los medios es muy amplia"

"Las noticias más interesantes para los medios son las que tengan que ver con temas salariales y/o conflictos laborales"

"Los temas positivos parecen tener mucho menos interés y si se destacan suele ser como respuesta a un conflicto"

"Podemos decir, por tanto, que interesan mucho los datos en general (números, porcentajes...) y los temas conflictivos, relacionando casi siempre nuestro objeto de trabajo con situaciones de riesgo".

Por otra parte, las noticias que, en función de la línea editorial del medio, pueden servir de punta de lanza para ahondar en cuestiones difíciles:

"Para ellos tienen más interés las que, en función de la línea editorial del medio, puedan servir de punta de lanza para ahondar en cuestiones espinosas o las llamativas por su fondo (p.ej. el conflicto en la Justicia, temas de Interior,...)"

"Cada medio tiene su línea editorial y por ello es muy importante ajustar el contenido de cada comunicación al tipo de medio al que va dirigida".

Los periodistas de medios, por su parte, señalan que la información que tiene más interés para ellos es aquella que no es facilitada explícitamente y a la que hay que saber acceder. También se apuntan otros criterios directamente relacionados con la noticiabilidad de los acontecimientos como es la actualidad:

"Normalmente las informaciones facilitadas por los responsables de prensa son excesivamente oficialistas. Casi nunca coincide lo que destaca el gabinete de prensa oficial con lo que finalmente aparece publicado. Esa suele ser la infor- 
mación de mayor interés para el medio, aquella que no ha sido destacada por el gabinete de prensa, y que hay que saber encontrar".

"Las informaciones que tienen más interés para nuestra agencia son las que se refieren a los temas de más candente actualidad: sobre todo de tipo político y económico".

\section{Valoraciones y apreciaciones sobre las relaciones entre fuentes y medios}

Para conocer cómo se valoran y aprecian las relaciones habituales entre periodistas de fuentes y periodistas de medios, se plantearon tres preguntas en la primera ronda del Delphi:

\subsection{Valoración de las relaciones entre los gabinetes de prensa y los medios}

En casi la totalidad de los casos, los expertos han respondido que las relaciones son buenas, incluso muy buenas; sólo puntualmente se ha considerado que las relaciones son normales, cordiales o ambivalentes. Se insiste, por parte de los periodistas de fuentes, en el hecho de que existe una mutua dependencia y en que este resultado se consigue con el tiempo, ejerciendo la transparencia y la claridad:

"Las relaciones son buenas. Salvo contadas excepciones, no tenemos "conflictos" con la prensa, ni tenemos que gestionar con frecuencia situaciones de crisis"

"Buenas, quizá óptimas. Sabemos que nos necesitamos mutuamente, que estamos obligados a convivir"

"Sin llegar a óptimas, sí podemos decir que son buenas".

"Buenas si ambas partes son profesionales y se entiende que cada uno está haciendo su trabajo".

"Periodista de medios y periodista de gabinete, aunque con intereses a veces opuestos, son también colaboradores. El medio quiere noticias, y la institución quiere que sus noticias lleguen al gran público, se necesitan.

"Las relaciones con los profesionales de los medios yo las señalaría como normales, cordiales, por lo general buenas".

"Es una relación ambivalente de amor-odio, de necesidad a su pesar. Quiero decir, que los periodistas saben que les facilitamos mucho las cosas, les damos información y les permitimos acceder a directivos".

Los periodistas de medios suscriben que, en general, las relaciones son buenas por las mismas razones aducidas anteriormente, si bien un profesional de los medios apuntó el hecho de que la independencia de criterio que debe imperar en los medios, puede dificultarlas:

"Generalmente regulares por perseguir metas diferentes que van de la información a la publicidad. Este tipo de relación es sin embargo saludable si tenemos en cuenta la necesaria independencia del periodista, algo que, aunque en tela de juicio, sigue estando presente en este trabajo"

Cuando los profesionales son preguntados a apropósito de si cambia la valoración de las relaciones según el tipo de noticias, el tipo de medio o según el periodista, los expertos respondieron en distintos sentidos. La opinión mayoritaria se inclina hacia el hecho de que sí cambia esta valoración sobre todo, en función de quién sea el periodista y, secundariamente, en función del tipo de noticia y su interés, o el medio: 
"La valoración está directamente relacionada con la persona del periodista"

"Sí. La predisposición del periodista es determinante".

"Por supuesto que sí. Quizá no cambie tanto según el tipo de noticia, pero seguro que sí influye el tipo de medio".

"Varía según el tema de la noticia y el tipo de medios. Los medios económicos son tal vez los más reticentes a tener intermediarios".

"Evidentemente, los medios tienen a veces una ideología política y unos intereses económicos que también cuentan. Unos apreciarán más a una institución que a otra, o un tipo de noticias más que otras".

"Cuando hay una noticia de peso, la relación es más fácil, tienes que vender y convencer menos. La noticia se abre paso por sí sola".

"Naturalmente del tipo de noticias y de su interés general, aunque influya el periodista y el medio".

Un pequeño grupo de periodistas opinó, sin embargo, que no cambia ni en función del medio ni en función de la persona. En cualquier caso, sí se resalta la importancia que tienen las relaciones personales para ambos:

"Pero la realidad es que esas relaciones personales a las que aludía son fundamentales, así como la posición ideológica del medio y la fuente. En organizaciones con alguna connotación ideológica, del tipo de sea, se aprecia con más claridad la "cercanía" y más fácil entrada en medios afines y, por el contrario, la ignorancia persistente (o el "mal trato") de otros medios".

"Es posible que, teniendo en cuenta que éste es un trabajo de relaciones interpersonales, se produzca alguna situación con algún periodista en concreto que, por su específica actividad o por su personalidad, pueda derivarse en algún roce o falta de entendimiento, pero son excepciones que confirman la regla de la cordialidad".

Respecto a la respuesta que dieron los periodistas de gabinete sobre si consideran que los medios en general privilegian a unas fuentes u organizaciones sobre otras y que criterios son los que priman, contestaron en general que la discriminación por parte del medio existe y que son varios los parámetros que influyen. Los expertos apuntaron principalmente a la afinidad y familiaridad que exista con el periodista de la empresa, al tipo de fuente u organización de que se trate en cada caso y al tipo e importancia de la noticia.

"Si los periodistas saben que las personas que llevan el Gabinete de Prensa contestan siempre, a tiempo y amablemente, influye bastante. Pero lo que prima por encima de todo son los intereses políticos del medio".

"El prestigio y fiabilidad de la organización y, en especial, de su máximo responsable".

"Obviamente, sí. Hay fuentes y organizaciones a las que les cuesta mucho entrar en los medios aunque puedan ser objetivamente un buen referente para la noticia y, al contrario, otras fuentes cuya entrada es casi automática por el prestigio que han atesorado, por lo que representan, por lo grandes que son... Creo que en general depende de la credibilidad de la fuente, del tipo de organización". 
"Definitivamente, en mi opinión los medios de comunicación establecen un sutil balance entre el tipo de fuente u organización y el tipo de noticia, privilegiando en primer lugar el "tamaño" de la fuente, que puede ser compensada si el tipo de noticia tiene rango superior o puede generar un mayor impacto".

"Probablemente, pero eso se debe a la experiencia de cada medio con esa fuente: si es creíble o si le ha respondido bien en el pasado".

"El tipo de noticia cuenta mucho, y, por descontado, la organización".

"Todo influye. En nuestro caso, creo que por el tipo de noticia y por la propia institución".

En segundo lugar, se señaló el peso publicitario de la empresa y marca que representan, como también se ha señalado en otros estudios recientes (MACIÁ, C. y HERRERA, S., 2010a y 2010b).

"Aunque suele influir sobre todo la importancia o magnitud de la noticia, algunos medios sí privilegian a unas fuentes sobre otras, en especial cuando la fuente realiza importantes inversiones publicitarias".

"Cuando el peso es más ambiguo o subjetivo, pesan también otras situaciones: la familiaridad con el periodista, la regularidad de la fuente, la originalidad del tema, el peso publicitario, el peso de la marca, etc."

Solo en un caso se obtuvo una respuesta negativa:

"En general, no. Aunque siempre, y como en todo, hay afinidades o complicidades"

Esta misma discriminación fue corroborada por los periodistas de medios que aducen las distintas afinidades e intereses en juego.

"Lo creo firmemente...Pero las noticias de verdad se filtran a personas concretas o medios determinados por razón de todo tipo de afinidades"

"Sin duda hay una discriminación según la procedencia u orientación del medio de comunicación. Los gabinetes de prensa seleccionan a los medios y a los periodistas según sus intereses"

\section{Prácticas profesionales y rutinas de selección, jerarquización y ubicación de in- formaciones}

Interesaba reflexionar sobre las prácticas y rutinas profesionales que son propias de los periodistas tanto de fuentes como de medios (RodRIGo AlsinA, M., 1993). A estos efectos se diseñó un segundo eje para el Delphi basado en tres preguntas, que han permitido detectar aquellos hábitos y actitudes que atañen a la selección, jerarquización y ubicación de noticias, que pueden comprometer el flujo de informaciones entre las organizaciones, instituciones o empresas, y los medios.

\subsection{Selección}

Respecto al proceso a partir del cuál se seleccionan las noticias dentro de los medios, interesaba conocer cuál es el criterio que a juicio de los profesionales prevalece, en especial si éste se inclina hacia el peso que puedan tener las fuentes y su credibilidad, o 
hacia el valor intrínseco de la noticia, es decir, actualidad, novedad, impacto, etc. ${ }^{4}$. Las respuestas de los profesionales se decantan, en primer lugar, por dar más importancia al tipo de noticia y los valores noticiosos del acontecimiento (relevancia, novedad, actualidad, interés, impacto):

"El criterio es el de la relevancia política y periodística".

"Que sea novedad, que anuncie algo nuevo y que pueda tener interés para los lectores".

"En mi opinión, el criterio se basa en la actualidad de la noticia y en el impacto que dicha noticia supone para la Compañía".

"El impacto mediático, lo que ellos puedan considerar como tal, lo que prevalece en la selección de noticias. Influye más el tipo de noticia".

"El periodista es un vendedor de actualidad. Y su público compra un tipo determinado de noticias. No compra todo. Por eso, el primer criterio de selección es que guste o interese a su audiencia".

"El tipo de noticia primero. Entre dos noticias del mismo peso, la fuente también es importante".

Y, en segundo lugar, el tipo de fuente, por su especialización y credibilidad o a ambas cosas:

"Primero el tipo de fuente, si se dedica al ámbito político o de la economía tiene mejor acogida, y después, el valor de la noticia".

"[...] no hay una forma prevalente de trabajar desde los medios en relación con las fuentes y que más bien lo que se da es una combinación de todas ellas. En ocasiones, interesa el tema, la noticia en sí. Otras veces, es la credibilidad de la fuente la que arma la información".

Los profesionales de los medios ante la misma la pregunta contestaron en dos sentidos diferentes: por una parte, que prevalecen principalmente la actualidad y el interés de la noticia más que la fuente (aunque apuntan que la credibilidad de dicha fuente es importante); y por otra, que la fiabilidad de la fuente es lo más importante.

\subsection{Jerarquización}

Respecto al proceso a partir del cuál se jerarquizan las noticias dentro de los medios, interesaba conocer cuál es el criterio que a juicio de los profesionales prevalece; en especial, si éste se inclina hacia el peso que puedan tener las fuentes o hacia el valor intrínseco de la noticia ${ }^{5}$. Los periodistas de fuentes declararon que ambos criterios son importantes a la hora de jerarquizar las noticias dentro de los medios:

"El tipo de noticia"

"El tipo de fuente"

${ }^{4}$ La pregunta formulada en este caso fue: ’En su opinión, ¿qué criterio prevalece en los medios a la hora de seleccionar las noticias que Vds. les envían? ¿Qué influye más en ese criterio: el tipo de fuente u organización, o el tipo de noticia?".

${ }^{5}$ En esta ocasión la pregunta formulada fue: "En su opinión, ¿qué criterio prevalece en los medios a la hora de jerarquizar las noticias que Vds. les envían? ¿Qué influye más en ese criterio: el tipo de fuente u organización, o el tipo de noticia?". 
"Influye mucho el tipo de organización pero también la importancia de la noticia". "El tipo de noticia. De nuevo, tienen más peso las de carácter económico o estratégico, y les dan mucha más importancia si la tienen en exclusiva".

"A la hora de jerarquizar se tiene más en cuenta el tipo de fuente".

"Mantengo que la fuente $\mathrm{u}$ organización tiene un peso específico superior que el tipo de noticia".

Y también, criterios periodísticos relacionados con el impacto de la noticia en la audiencia, el peso e importancia de la misma y el interés político y social, así como el hecho de que se trate de temas conflictivos, sin olvidar la competencia con otras noticias dentro del medio:

"Siempre el peso de la noticia, el impacto en los lectores".

"Las noticias las jerarquizan por el interés político y social que puedan despertar en la ciudadanos y dependiendo también de los criterios periodísticos propios de la empresa informativa".

"En nuestro caso priman, como decía, los temas conflictivos, incluso diría que cuanto más lo sean mejor y esta es una tendencia difícil de modificar"

Ante la misma pregunta formulada a los periodistas de medios, éstos contestaron en dos sentidos diferentes: 1) se apunta que la fuente (fiabilidad) es lo primero y después el criterio de actualidad; 2) que el criterio de jerarquización viene dado por el interés, alcance y trascendencia de la noticia.

\subsection{Ubicación}

Respecto al proceso a partir del cuál se ubican las noticias dentro de los medios, interesaba conocer cuál es el criterio que a juicio de los periodistas de fuentes prevalece, en especial si éste se inclina hacia el peso que puedan tener las fuentes o hacia el valor intrínseco de la noticia ${ }^{6}$.

En opinión de los expertos consultados, la ubicación de las noticias depende, de manera rotunda, del tipo de noticia de que se trate en cada caso. Enviar la noticia a la sección correcta, con una presentación del contenido en forma sintética y llamativa resulta de utilidad, aunque a la hora de decidir en qué lugar situarlas, priman los criterios periodísticos y los valores noticiosos:

"Pienso que influye más el tipo de noticia".

"Del tipo de noticia y de la persona que la protagoniza".

"Prevalece el criterio periodístico que fija cada publicación".

"A los medios hay que enviarles las noticias a la sección correcta donde tenga cabida, si es de economía, política, cultura o ciencia. Después, la organización tiene mucha importancia, su prestigio, pero por descontado, el valor social de la noticia es muy destacado. A cuanto más público le afecta o interesa, más valor tiene para los medios".

"El tipo de noticia, el impacto en la audiencia, la novedad, la cercanía".

${ }^{6}$ En esta ocasión la pregunta formulada a los profesionales fue: "En su opinión, ¿qué criterio prevalece en los medios a la hora de ubicar en diferentes lugares las noticias que Vds. les envían? ¿Qué influye más en ese criterio: el tipo de fuente u organización, o el tipo de noticia?”. 
Ante la misma cuestión, los periodistas de medios contestaron en dos sentidos diferentes: algunos profesionales declaran que ubican las noticias que reciben según el contenido; otros periodistas respondieron que ubican las noticias según la fuente y la presentación de la noticia.

\section{Credibilidad de la fuente y otros criterios que comprometen las rutinas profe- sionales}

Estas observaciones relacionadas con las rutinas que pueden comprometer el flujo de informaciones entre periodistas de fuentes y medios, se corroboraron en la segunda ronda del Delphi. Interesaba entonces profundizar en lo relativo a la credibilidad de las fuentes, toda vez que éste había sido un tema aparecido de manera recurrente en las declaraciones de los expertos. Esto se ha hizo a partir de tres nuevas preguntas formuladas en la segunda ronda:

1. "En su opinión ¿a qué afecta más la credibilidad de la fuente?: ¿A la selección de la noticia para ser publicada? ¿A la jerarquización respecto a otras noticias? ¿O a la ubicación de la noticia en el propio medio? “

La mayor parte de los expertos consultados contestaron que en lo que más influye es en la selección y secundariamente en la jerarquización de la noticia o en ambas:

"A la selección y, en menor medida, a la jerarquización. Lo más importante para la organización es aparecer en los medios, ser visibles ante la sociedad"

"Creo que directamente a la selección de la noticia para ser publicada, ya que, una vez confirmada por parte del periodista la credibilidad de la fuente, la ubicación o jerarquización sería una fase posterior"

2. “¿Piensa que para que su noticia adquiera una buena jerarquización y ubicación en el medio hay que enviarle al periodista una información bien acabada para ser publicada, y que llegue a la sección correcta?"

Puede considerarse que la totalidad de los periodistas consultados están absolutamente de acuerdo en que cuanto más acabada se proporcione la noticia, mejor será para su publicación, e incluirla en la sección correcta es imprescindible. También señalan que es importante el tratamiento y redacción que se proporcione: textos cuidados, claros y concisos. Esto se hace imprescindible para los profesionales porque en ello recae el buen nombre o la imagen de marca de la organización en cuestión.

"Todo lo que sea facilitar al máximo la labor del periodista nunca caerá en saco roto. Además, creo que es una obligación de la fuente elaborar textos cuidados, claros y concisos. Está en ello el buen nombre o la imagen de marca de la organización en cuestión".

"La información, ya sea una nota de prensa o un dossier, tiene que estar redactada y presentada de acuerdo con las expectativas que tiene cada medio. Lo ideal sería personalizar la noticia para cada medio. Como eso es casi imposible, hay que encontrar un denominador común que responda a criterios periodísticos".

"Absolutamente. Estoy de acuerdo con las dificultades que se le plantean al periodista, por lo que todo lo que pueda contribuir a facilitar su trabajo y permita una mejor selección y jerarquización, mucho mejor". 
3. “¿Cree que priman también otros motivos relacionados con afinidades ideológicas, económicas o coyunturales del medio?"

Respecto a esta pregunta, las respuestas aparecen divididas a partes casi iguales entre los aquellos que opinan que sí y los que opinan que no. Llama la atención que incluso algunos de los que responden en sentido negativo, añaden que siempre hay que tener en cuenta este tipo de condicionantes, pero no creen que sean decisivos.

"Por supuesto. Sin ningún género de dudas".

"En algunos casos si, especialmente cuando se trata de temas polémicos o de mucha trascendencia".

"Sí, creo que las afinidades juegan siempre un papel importante, en general, a la hora de valorar y publicar las informaciones".

"También influyen, pero pesa sobre todo la credibilidad de la fuente, que es una combinación de la experiencia de relación con esa organización y con la persona que actúe como portavoz habitual".

"En principio, prefiero pensar que no, aunque en determinadas circunstancias y bajo determinadas premisas, pudiera contemplarse. Sin embargo, me resisto a definir estos criterios como regla básica".

"Creo que no es así".

"Siempre hay que tener en cuenta estos condicionantes pero no creo que sean decisivos en la gestión de la relación con los medios".

"Determinadas noticias se eligen por el posicionamiento ideológico del medio y también, por los propios intereses del grupo de comunicación al que pertenezca".

\section{Afinidades ideológicas, políticas y económicas en la valoración de las relacio- nes entre fuentes y medios}

De todos es sabido que la autonomía periodística pasa por la independencia respecto a los poderes políticos y económicos. En este sentido, interesaba conocer en qué términos se lleva a cabo y cómo condiciona el ejercicio de la profesión. Estudios anteriores (CANEL et al.: 2000) han mostrado que si bien la presión política se hace siempre notar, ésta no es decisiva a juicio de los propios profesionales. La propiedad del medio y la identidad de los anunciantes se sitúan por encima de las presiones políticas.

En la primera ronda del Delphi, respuestas de los periodistas consultados apuntaban al hecho de que los medios, en general, privilegian unas fuentes u organizaciones sobre otras, y que dicha discriminación existe. Aunque son muchos los parámetros que influyen, principalmente se refirieron a dos: la afinidad y familiaridad que exista entre periodistas de fuentes y medios y, la más importante, en la que casi todos coincidieron, el peso publicitario de la organización en el medio y la preeminencia de la imagen o marca que la organización representa.

En la segunda ronda, ante la pregunta: “¿Considera que la afinidad política es el criterio de mayor importancia a la hora de valorar las relaciones con los medios? ¿O es el interés económico?", se confirma que la valoración de las relaciones entre empresas, organizaciones e instituciones y medios, depende de factores como el criterio económico, el interés de la noticia, la afinidad política y el perfil de la empresa informante por este orden de importancia en cuanto al volumen de las respuestas. 
"Creo que el interés económico está incluso por encima de la afinidad política"

"Creo que el interés económico es un factor condicionante básico".

"Depende: en la información institucional pesa más la orientación política del medio; en la empresarial, la económica".

"No. Creo que es el interés de la noticia, sin más".

"Ambos son importantes, pero la clave siempre estará en la importancia de la noticia".

"El interés económico primero pero la afinidad política seguida inmediatamente". "La afinidad política".

"La afinidad política es un criterio muy relevante, más que el interés económico".

"La "afinidad política", entendida en un sentido amplio, tiene una ponderación mayor que el interés económico en el largo plazo. Tal vez en el corto la publicidad pueda mover algunas voluntades".

"Aunque el peso publicitario puede llegar a influir en el reparto de espacio, no creo que sea tan decisivo. La reputación de una compañía, su credibilidad y las relaciones del departamento de comunicación con los periodistas de un medio son más importantes".

Ante la pregunta "¿Esta valoración cambia según el tipo de noticias, el tipo de medio, o la persona del periodista?", las respuestas fueron muy dispersas, repartiéndose a partes casi iguales entre aquellos profesionales que opinaron que sí cambia, los que opinaron que no cambia, los que opinaron que cambia según el tipo de medio y el tipo de noticia, y aquellos que pensando que si cambia, consideran que es atribuible a múltiples factores:

"El tipo de medio es fundamental"

"Ante todo, según el tipo de noticia"

"Se matiza, pero no cambia"

"El tipo de noticia primero y después el tipo de medio"

"La valoración varía según la noticia y el medio, aunque la afinidad con el periodista puede salvar esas dos valoraciones"

"Hay medios en los que la afinidad ideológica juega un papel más importante. Digamos que 'se colocan' las informaciones más fácilmente; en otros medios cuesta más trabajo conseguirlo. En estos últimos, sólo cuenta la propia noticia, que tenga interés en sí misma. En ocasiones también puede ocurrir incluso al contrario: la lejanía ideológica puede influir en que determinadas informaciones, que son objetivamente interesantes, no se publiquen"

"La valoración cambia en función del tipo de medio y también de la persona del periodista más que en función del tipo de noticia"

"Sin duda, en noticias de aspecto político pudiera tener este criterio un mayor peso, teniendo en cuenta, además, que determinados medios se han posicionado manifiestamente favorables a criterios políticos de uno u otro signo"

"Obviamente, en la medida en que la gestión de la relación de una compañía con cada medio y con cada periodista es distinta" 


\section{Hábitos y actitudes que comprometen las prácticas profesionales}

En la primera ronda del Delphi se cuestionaba a los periodistas a propósito de aquellos hábitos o actitudes que pueden comprometer las prácticas profesionales respecto a la selección, jerarquización y ubicación de las noticias. En la tercera y última ronda, se profundizó sobre estas cuestiones.

\subsection{Preferencia y uso de géneros periodísticos}

Los periodistas de gabinete fueron invitados a expresar si negocian sus preferencias por géneros periodísticos, cuando envían la información a los medios, a partir de la siguiente pregunta: “¿Qué importancia tiene para su empresa o institución que los medios elijan los distintos géneros, con independencia del contenido de las informaciones que usted les ofrece?".

Los periodistas contestaron que los géneros en que se publica la información que envían a los medios, tienen mucha importancia cuando se trata sobre todo de artículos o editoriales, secundariamente cuando se trata de entrevistas o crónicas; y tienen menos importancia cuando se trata de reportajes y noticias. Es decir las fuentes otorgan más importancia a que sus informaciones aparezcan publicadas en géneros de opinión, secundariamente en géneros interpretativos y en tercer lugar en géneros informativos.

Preguntados los periodistas de fuentes a propósito de “¿En qué casos acuerdan con los medios los géneros en que se publican las informaciones enviadas?", se obtuvieron respuestas en distintos sentidos. El sentir mayoritario se inclina hacia el hecho de que no se acuerda por distintas razones: porque es el medio o su redactor jefe el que decide, por ser una fuente institucional que excluye dichas prácticas y por la propia naturaleza de las informaciones que proporcionan:

"El Departamento de Medios de Comunicación del Senado es institucional, y no solemos realizar esta práctica".

"Casi nunca. Es él o su redactor jefe quién decide".

"Cuando se trata de informaciones o propuestas que no están sujetas a la regulación de hechos relevantes o que deban ser comunicados a la Comisión Nacional del Mercado del Valores".

En algún caso, los periodistas de fuentes manifiestan que sí se acuerdan los géneros e indican que esto es así principalmente cuando se trata de géneros de interpretación como la entrevista, que además resulta el más deseable:

"En el caso de las entrevista, el resto puedo sugerir, nunca pedir un género u otro".

"Siempre que se pueda. No es lo mismo un artículo de opinión que el editorial del propio periódico. Me gusta el formato entrevista, más que incorporar información de la Compañía en forma de noticia".

Respecto a cuál es el grado de satisfacción con relación a la elección de géneros que hacen los medios, se obtuvo una respuesta contundente en sentido positivo.

"Si. Si son informaciones interesantes le dedican el espacio y el tratamiento adecuado".

"En general sí, pues normalmente el tipo de noticia o el momento de su difusión te marca la elección del género". 
"Si suelen distinguir acertadamente cuando una información debe ser tratada como reportaje, crónica, artículo de opinión, etc.".

\subsection{Autoría y protagonismo de las informaciones publicadas como consecuencia de las relaciones entre periodistas de fuentes y medios}

Los periodistas de fuentes fueron invitados a manifestar sus acuerdos con los medios sobre la autoría más conveniente para la publicación de sus informaciones que pueden ir firmadas y/o sobre los personajes que hayan de proponerse como protagonistas de esas informaciones y su grado de satisfacción al respecto.

A la pregunta "¿Qué importancia adquiere para su empresa o institución quién firme la información publicada?", los periodistas de gabinete manifestaron otorgar mucha o bastante importancia a que sea un periodista del medio o la propia institución quién firme la información, mientras que manifiestan que es menos importante para ellos que sea un colaborador externo o que aparezca sin firma.

Respecto a la importancia otorgada a quienes sean los protagonistas de las informaciones enviadas a los medios para ser publicadas, las respuestas de las fuentes señalan que para ellos es muy importante que sea un directivo de su institución o empresa y, en segundo lugar, que sea un líder de opinión del sector. Menos relevancia conceden a que sean los miembros de la empresa o institución o el público externo quién protagonice. Es decir, como cabría esperar, las fuentes están interesadas en dar visibilidad a sus propios directivos o líderes.

Preguntadas las fuentes por su grado de satisfacción respecto a cómo los medios visibilizan este protagonismo y fijan la autoría de la información, las respuestas mayoritarias se muestran en sentido positivo.

"Sí. Son profesionales, objetivos, y de buen trato personal".

"Habitualmente si, existen periodistas que siguen la información del Senado y al 'conocer' la Cámara saben quienes son los protagonistas de la información".

"En nuestro caso no elegimos quién firma las informaciones, sí quién las protagoniza, por tanto estamos satisfechos en este punto y en el primero (quién firma) depende de las ocasiones".

Solo en dos ocasiones se obtuvieron respuestas de carácter menos definido:

"En definitiva lo que importa es que se publique y que la información sea correcta, no quien firme el artículo".

"La Institución trata temas de relevancia social que despiertan opiniones de muy distinta orientación. Esto hace que aparezcan protagonistas y firmantes de informaciones que, en muchos casos, no están suficientemente cualificados o no son las personas más relevantes en el ámbito concreto de la noticia que se está tratando".

\subsection{Elección de las secciones en las cuales se ubican las informaciones publicadas} Los medios privilegian determinadas secciones en función de las informaciones de que se trate en cada caso, interesaba conocer cómo se valoran las correspondencias entre las secciones elegidas por los medios y las deseadas por las fuentes para la publicación de sus informaciones. Las preguntas formuladas a los profesionales invita- 
ban a manifestar sus acuerdos respecto a las secciones habitualmente elegidas por los periodistas de medios.

A las preguntas " $¿$ Qué importancia le concede a la ubicación de sus informaciones en las secciones que los periodistas eligen para publicarlas? ¿Y corresponde la sección elegida al perfil de la información entregada?", los profesionales expresaron que sí resulta importante para ellos y que en general hay bastante coincidencia.

"Generalmente, nuestras informaciones se publican en la sección adecuada"

"Mucha"

"Sí, suele coincidir el perfil de la información con la sección, no se elige"

"Suele ser en las secciones de economía o empresas, que suele ser lo adecuado"

A la pregunta "¿Cuál es la sección o secciones habitualmente elegida(s) por los medios y deseada(s) para sus informaciones por su empresa o institución?", los periodistas contestaron que la sección preferente es la portada aunque no es la habitual para la publicación de sus informaciones. En segundo lugar se señala que la sección económica y, en tercer lugar, autonómica y local y ciencia y tecnología. Sin embrago, estos profesionales declaran que existen algunas discrepancias respecto a la elección de secciones que habitualmente hace el medio para publicar sus informaciones, siendo las más frecuentes economía y autonómica y local.

\subsection{Estructura del discurso en la que se integra la información de fuentes}

Finalmente, se invitó a los profesionales, en la tercera ronda, a concluir sus apreciaciones sobre la estructura del discurso en el que se integran más usualmente las informaciones brindadas a los medios. Para ello, se ha distinguido si en dicho discurso se hace referencia a "lo que se dice", "lo que se hace" o "lo que ocurre", a fin de contrastar estas respuestas con los resultados obtenidos en el Análisis de contenido de los periódicos estudiados en la otra parte de la investigación ${ }^{7}$. Las respuestas de los profesionales muestran que, en su opinión, el discurso sobre su institución o empresa se centra, casi en la mitad de los casos $(42,5 \%)$, sobre "lo que se dice" y en mucha menor proporción sobre "lo que ocurre" $(29,5 \%)$ y "lo que se hace" (28\%). Es decir se trata de un discurso esencialmente declarativo, y secundariamente centrado en el acontecer y en el hacer.

\section{Conclusiones}

De este estudio Delphi se pueden extraer estas conclusiones principales:

- Respecto al flujo de informaciones entre periodistas de fuentes y medios, sobresale la importancia otorgada a las relaciones personales, sostenidas cada vez más sobre las nuevas herramientas de comunicación como el correo electrónico, que se suma al teléfono como instrumento principal de relación. Estas relaciones personales resultan clave para las fuentes a la hora de lograr una mayor presencia en los medios, a lo que hay que hay que sumar otros criterios rela-

\footnotetext{
${ }^{7}$ En el análisis de contenido pudimos comprobar que las piezas o registros informativos cuya referencia estaba centrada en "lo que se dice" alcanzaba el $45 \%$, mientras en el resto se distribuían casi por igual las referencias a "lo que se hace" (28\%) y "lo que ocurre" (27\%).
} 
cionados con los valores que debe presentar cualquier acontecimiento para llegar a ser noticia: interés, importancia del tema y credibilidad de la fuente. Una buena gestión de estas relaciones, con las herramientas adecuadas, permite un buen volumen de mutua respuesta, más frecuente en los medios especializados que en los generalistas y agencias.

- La percepción de las fuentes respecto a lo que supone interés preferente para los medios, apunta hacia los temas dificultosos que suponen conflicto, crisis y, en general, carácter negativo. Se confirma el adagio vigente en la profesión: bad news, is good news. Sin embargo, los profesionales de los medios hacen alusión a criterios de noticiabilidad, como la actualidad, sin olvidar el hecho de bucear en los acontecimientos e ir más allá de la oficialidad.

- La valoración de las relaciones entre periodistas de fuentes y medios es positiva en general, aunque se apuntan factores que intervienen en ella, como la propia persona del periodista y la familiaridad o afinidad que les une o el hecho de que se discrimina una $u$ otra fuente en función de quién se trate en cada caso, la empresa o el peso de la marca que representa.

- Las rutinas productivas, selección, jerarquización y ubicación de noticias, se asientan sobre la base de los valores noticiosos declarados: relevancia, novedad, actualidad, interés, impacto, además de la fiabilidad de la fuente, la cuál se presenta como decisiva para que los acontecimientos aparezcan reseñados como noticias en los medios.

- Aparecen posturas diversas entre los profesionales, a favor y en contra, cuando se trata de valorar si las afinidades ideológicas, económicas o coyunturales afectan a las rutinas profesionales.

- Las fuentes declaran abiertamente su interés por colocar sus informaciones principalmente en géneros de opinión (en segundo lugar en géneros interpretativos y en tercer lugar, informativos), lo que intentan negociar en la medida de lo posible.

- Conseguir la máxima visibilidad para la empresa o institución que representan forma parte de las aspiraciones de los periodistas: dar la palabra a sus directivos o líderes de opinión y tener un lugar preeminente en el medio (por ejemplo, la portada), si bien valoran satisfactoriamente la labor que, en este sentido, llevan a cabo los profesionales de los medios.

En resumen, es en las rutinas productivas del periodismo en donde se libran los ajustes necesarios para hacer compatibles afinidades e intereses diversos, económicos, comerciales, ideológicos, políticos o meramente coyunturales, más o menos reconocidos por los profesionales.

Las manifestaciones de los profesionales tanto de fuentes como de medios son coincidentes en que el discurso noticioso es esencialmente declarativo, frente al discurso de los acontecimientos o de los actos que los agentes ponen en juego a propósito de esos acontecimientos. Dicho en otros términos, se trata más de dar cuenta de lo que se dice que de averiguar lo que acontece y dar respuesta a la exigencia social de la verdad, en el proceso de producción y reproducción de los conocimientos compartidos socialmente a partir de la práctica cotidiana de los medios. Estos resultados 
son enteramente coincidentes con los aportados por el Análisis de contenido en la otra parte de la investigación respecto a la estructura del discurso en la que se integra más usualmente la referencia a la propia institución o empresa en los medios de comunicación (el $45 \%$ de las piezas o registros informativos estaba centrada en "lo que se dice", el $28 \%$ en "lo que se hace" y el $27 \%$ en "lo que ocurre") (Cf. PIÑUEL, J.L. y GAITÁN, J.A., 2010).

El que se trate de un discurso esencialmente declarativo corrobora el planteamiento de la investigación al cuestionarse sobre las condiciones de producción del mensaje informativo con relación a la verdad y a los ejes sobre los que se articula en el discurso de la prensa. Estos resultados ilustran el hecho de que la producción de comunicación es un proceso de mediación basado en gran medida en ofrecer veracidad y credibilidad, es decir legitimidad, pero no se puede olvidar que cuando en los medios la noticia no es el acontecimiento sino la noticia del acontecimiento, estamos ante la lógica de la simulación (BAUDRILLARD, 1984) que desprovee al signo de valor sígnico y a la realidad misma de referencia real.

\section{Referencias bibliográficas}

BAUDRILLARD, Jean (1984): Cultura y simulacro. Barcelona, Kairós.

CANEL, María José; RODRÍGUEZ ANDRÉS, Roberto y SÁNCHEZ ARANDA, José Javier (2000): Periodistas al descubierto. Retrato de los profesionales de la información. Madrid, CIS.

FRANKENBERG, Lorena y LOZANO, José Carlos (2010): "Rutinas, valores y condicionantes en la producción de la noticia: el testimonio de cuatro directores de medios informativos en Monterrey, México", en Comunicación y Sociedad, vol. XXIII, $n^{\circ}$ 1, 2010, pp. 175-204.

GAITÁN, Juan Antonio y PIÑUEL, José Luís (1998): Técnicas de investigación en comunicación social. Elaboración y registro de datos. Madrid, Síntesis.

GAITÁN, Juan Antonio, PIÑUEL, José Luís, CÁCERES, Ma Dolores, LOZANO, Carlos (2009): "Las claves de sentido sobre la comunicación social en la agenda mediática del discurso hegemónico de los diarios españoles de referencia dominante", en Trípodos, Número extra, Vol. 1, pp.55-65.

HUMANES, María Luisa (2007): "La investigación sobre la profesión periodística en España", en Sphera Publica, Número Especial, pp. 173-193.

MACIÁ, Carlos y HERRERA, Susana (2010a): "La excelencia informativa: dilemas éticos y retos profesionales", en Cuadernos de Periodistas, n 19. Madrid, Asociación de la Prensa, pp.66-95.

MACIÁ BARBER, Carlos y HERRERA DAMAS, Susana (2010b): "La deontología periodística: praxis, disfunciones y retos desde la perspectiva de los profesionales de la comunicación en la Comunidad de Madrid (2006-2009)", en Comunicación y Sociedad, vol. XXIII, n ${ }^{\circ}$ 1, pp. 77-104. 
McCOMBS, Maxwell (1996): "Influencia de las noticias sobre nuestras imágenes del mundo", en BRYANT, Jennings y ZILLMANN, Dolf: Los efectos de los medios de comunicación. Investigaciones y teorías. Barcelona, Paidós, pp. 13-34.

ORTEGA, Félix (2008): "Periodistas: entre la profesionalidad y el aventurerismo", en MARTÍNEZ NICOLÁS, Manuel (coord.): Para investigar la comunicación. Madrid, Tecnos, pp. 225-239.

PIÑUEL, José Luis y GAITÁN, Juan Antonio (2010):"El discurso hegemónico sobre la verdad y la comunicación en la autorreferencia mediática en Prensa", en Revista Latina de comunicación social, 65. La Laguna, Tenerife, Universidad de La Laguna, pp. 572-594 (DOI: 10.4185/RLCS-65-2010-921-572-594)

RODRIGO ALSINA, Miquel (1993): La construcción de la noticia. Barcelona, Paidós.

TUCHMAN, Gaye (1983): La producción de la noticia. Estudio sobre la construcción de la realidad. Barcelona, Gustavo Gili. 